\title{
Chronic fatigue syndrome: comparing outcomes in White British and Black and minority ethnic patients after cognitive-behavioural therapy
}

\author{
Tom Ingman, Sheila Ali, Kamaldeep Bhui and Trudie Chalder
}

\section{Background}

Cognitive-behavioural therapy (CBT) is one of the most promising treatments for chronic fatigue syndrome (CFS). It is unclear whether CBT is effective for Black and minority ethnic (BME) groups.

\begin{abstract}
Aims
To assess the effectiveness of CBT in BME patients compared with White British patients presenting to a specialist CFS service.
\end{abstract}

\section{Method}

Data from 67 (19.0\%) BME participants and 285 (81.0\%) White British participants referred to a specialist CFS service in the UK were collected at baseline and after CBT treatment.

\section{Results}

Pairwise comparisons revealed that both BME participants and White British participants significantly improved on measures of fatigue severity $(P<0.001)$, physical functioning $(P<0.001)$ and work/social adjustment $(P<0.001)$.
Independent samples $t$-tests showed that BME participants improved despite exhibiting significantly higher baseline damage beliefs $(P=0.009)$, catastrophising $(P=0.024)$, all-or-nothing behaviour $(P=0.036)$ and avoidance/resting behaviour $(P=0.001)$, compared with White British participants.

\section{Conclusions}

To our knowledge, this study is the first to indicate that CBT is effective for treating CFS in a group of patients from diverse BME backgrounds.

\section{Declaration of interest}

K.B. is Editor of the British Journal of Psychiatry; he was not involved in the decision to publish this paper. T.C. receives royalties for self-help books on chronic fatigue.

\section{Copyright and usage}

(c) The Royal College of Psychiatrists 2016.
Chronic fatigue syndrome (CFS), also referred to as myalgic encephalomyelitis (ME), is characterised by severe, disabling and medically unexplained fatigue, which is not alleviated by rest and lasts for at least 6 months. ${ }^{1}$ Initial research suggested that CFS predominantly affected young White women. ${ }^{2}$ However, subsequent population-based research has indicated that there is a higher prevalence of CFS in Black and minority ethnic (BME) groups, ${ }^{3}$ with these groups experiencing more severe symptoms $^{4-8}$ and employing more unhelpful cognitive and behavioural strategies, including denial, hope, prayer and behavioural disengagement, compared with ethnic majority groups $5,9,10$ Despite this, there is no clear evidence that cognitive-behavioural therapy (CBT), one of the most promising treatments for CFS, ${ }^{11}$ is as effective in BME patients as in White British patients. ${ }^{10}$ This is concerning given suggestions that therapies such as CBT may be preferentially recommended for people who have a university education and for people of European/American cultural origins, ${ }^{12}$ and may therefore lack cultural sensitivity for people of BME origins. Research into depression and psychosis has shown that BME patients have poorer CBT outcomes, ${ }^{13-16}$ and require cultural adaptation for it to be effective. ${ }^{17,18}$ However, although this may be the case for psychiatric disorders, there is no evidence that this is true for functional disorders such as CFS. Further work is therefore required to establish whether UK BME patients with CFS differ from White British majority patients in their response to standard, non-adapted CBT treatment. The aim of the present study was to assess the effectiveness of CBT in treating CFS in BME patients in comparison with White British patients referred to a specialist CFS service.

\section{Method}

\section{Hypotheses}

(a) At baseline, BME participants will have significantly higher fatigue severity, poorer physical functioning, poorer work and social adjustment, and more unhelpful cognitive and behavioural responses compared with White British participants.

(b) The post-CBT improvement in fatigue severity, physical functioning and work and social adjustment will be significantly higher in the White British group compared with the BME group.

\section{Participants}

Participants were assessed at the Chronic Fatigue Research and Treatment Unit in London, UK, which is part of King's Health Partners. Participants were selected in accordance with Oxford criteria for diagnosing CFS. ${ }^{1}$ These criteria, described previously, ${ }^{11}$ require fatigue to be the main symptom, accompanied by significant disability, in the absence of an exclusionary medical or psychiatric diagnosis, with the exception of comorbid depression (e.g. psychosis, bipolar disorder, substance misuse, organic brain disorder, eating disorder). All participants were medically assessed by specialist clinic doctors to exclude alternative diagnoses. ${ }^{19}$ The treatments offered were CBT and graded exercise therapy. Process measures and outcomes were routinely collected from all participants. Participants were excluded if they were under 18 years of age, pregnant, housebound or receiving treatment other than $\mathrm{CBT}$ (such as graded exercise 
therapy or adaptive pacing therapy), had participated in the PACE trial $^{11}$ or did not start treatment.

Participants were classified as being either White British (i.e. those who described themselves as 'White British' or 'White English') or BME, defined in this study as 'a minority group or any group within any one country from which its cultural, racial, ethnic or national identity is different to the dominant identity (or to the one shared by the majority) in their country of residence.' ${ }^{3}$

\section{Design}

A prospective design was used, with hand-completed, selfreported questionnaires at pre-treatment (baseline) and posttreatment (discharge). Data were collected between November 2007 and May 2012. The independent variable was ethnic group: BME and White British. Outcomes of interest included fatigue severity, physical functioning, work and social adjustment, and cognitive and behavioural responses to symptoms.

\section{Treatment}

CBT techniques for CFS have been described previously. ${ }^{20}$ Participants received CBT from a clinical psychologist or CBT therapist. Clinicians were experienced in CBT and CFS, were guided by a standardised CBT manual and had regular individual clinical supervision. Participants typically received one session of CBT per fortnight, and each received approximately 12 sessions. Eleven participants $(3.1 \%)$ were treated by a trainee clinical psychologist, supervised closely by one of the more experienced therapists.

\section{Evaluation of treatment}

\section{Fatigue}

The 11-item Chalder Fatigue Scale, ${ }^{21}$ shown to be valid and reliable in CFS patients, ${ }^{22}$ was used to assess fatigue severity. Each item has four possible responses, scored from 0 to 3: 'less than usual' (0), 'same as usual' (1), 'more than usual' (2) and 'much more than usual' (3). The possible score range is $0-33$, with a higher score indicating a higher level of fatigue.

\section{Physical functioning}

Ten items from the Short-Form Health Survey ${ }^{23}$ were used to measure the extent to which various physical activities (e.g. 'carrying groceries' and 'climbing stairs') were limited by health. Each item has three possible responses: 'limited a lot' (0), 'limited a little' (5) and 'not limited at all' (10). The possible score range is $0-100$, with a higher score indicating greater physical functioning. This scale has been shown to have excellent validity and reliability in CFS patients. ${ }^{24}$

\section{Work and social adjustment}

The five-item Work and Social Adjustment Scale ${ }^{25}$ was used to measure impairment of day-to-day functioning (e.g. 'going to work' and 'home management'). Each item has eight possible responses, ranging from 'not at all impaired' (0) to 'severely impaired' (8), and produces a score between 0 and 40. A higher score indicates a lower level of functioning. The Work and Social Adjustment Scale has been shown to be valid and reliable in patients with CFS. ${ }^{26}$

\section{Cognitive and behavioural responses}

The Cognitive and Behavioural Responses Questionnaire $(\mathrm{CBRQ})^{27}$ was used to assess patients' cognitive and behavioural responses to symptoms. The 42-item scale consists of five cognitive subscales and two behavioural subscales. Four of the cognitive subscales assess how respondents interpret their symptoms: fear avoidance (e.g. 'I am afraid that I will make my symptoms worse if I exercise'), damage beliefs (e.g. 'the severity of my symptoms must mean that there is something serious going on in my body'), catastrophising (e.g. 'I will never feel right again') and embarrassment avoidance (e.g. 'the embarrassing nature of my symptoms prevents me from doing things'); the fifth cognitive subscale measures symptom focusing (e.g. 'when I am experiencing symptoms it is difficult for me to think of anything else'). The two behavioural response subscales assess avoidance/resting behaviour (e.g. 'I stay in bed to control my symptoms') and allor-nothing behaviour (e.g. 'I tend to do a lot on a good day and rest on a bad day'). Each CBRQ item has five possible responses, scored from 0 to 4 . Higher subscale scores indicate more unhelpful cognitions and behaviours. The CBRQ has been shown to be valid and reliable in patients with multiple sclerosis ${ }^{28}$ and CFS. ${ }^{29,30}$

\section{Ethics}

Confidentiality and the right to withdraw were explained to all participants. This study was part of a clinical audit of routinely collected data; therefore ethical approval was not required. An Audit and Service Evaluation Project Proposal Form was submitted and approved by South London and Maudsley's Psychological Medicine Clinical Academic Group Audit Committee and the clinical governance department, part of King's Health Partners.

\section{Data screening}

Before screening, the data-set comprised 519 participants, reduced to 405 after applying exclusion criteria. Of the excluded participants, $43.9 \%(n=50)$ were receiving treatment in addition to CBT, $23.7 \%(n=27)$ had a diagnosis other than CFS, $15.8 \%(n=18)$ did not start treatment, 7\% $(n=8)$ did not meet CFS criteria, $2.6 \%(n=3)$ did not complete any questionnaires, $2.6 \%(n=3)$ were pregnant, $2.6 \%(n=3)$ were housebound, $0.9 \% \quad(n=1)$ had participated in the PACE trial and $0.9 \%(n=1)$ had received treatment twice.

Of those who did not start treatment, $27.8 \%(n=5)$ did not reply to letters/phone calls, $27.8 \%(n=5)$ did not want CBT treatment, $16.7 \%(n=3)$ had moved abroad, $11.1 \%(n=2)$ had been referred for treatment elsewhere, $11.1 \%(n=2)$ did not attend their first session and 5.6\% $(n=1)$ did not have funding. Chi-square analysis and $t$-tests revealed that starters and nonstarters did not differ in terms of age, gender or ethnic group. The data were examined for errors, missing values and incomplete questionnaires. Participants with incomplete baseline questionnaires $(n=26)$ were removed from the data-set.

Participants who did not disclose their ethnicity, or defined themselves as 'British' or 'English' $(n=24)$, were recorded as missing. A repeated-measures analysis of variance revealed no significant interaction effect of time $\times$ group (missing, BME and White British), for fatigue severity $(F(1,227)=0.29, P=0.75$, $\left.\eta_{P}^{2}=0.00\right)$, physical $\eta_{P}^{2}=0.02$ ). Pro-rated data were used for participants who had $\leqslant 30 \%$ missing responses for each of the measures listed above (or for each subscale on the CBRQ). Pro-rating involved calculating the mean value of the item scores present and replacing the missing values with that score. ${ }^{11}$

Histograms and boxplots were visually inspected for normality and outliers, respectively. Normality was assumed if skewness was $<1$ and kurtosis $<3$. The assumption of normality was largely met, 
with the exception of positive skewness in post-CBT avoidance/ resting behaviour in the BME group, therefore analysis of this variable should be interpreted with caution. Obvious univariate outliers on boxplots were corrected by replacing these with values inside the normal range. Three cases with multivariate outliers were discarded, leaving 352 cases for analysis.

\section{Analysis}

The data were analysed using SPSS version 22. Statistical significance was defined as $P<0.05$. Group differences at baseline were tested using independent samples $t$-tests for continuous data and chi-square for categorical data. Hypothesis 1 was assessed using an independent samples $t$-test to compare groups on baseline cognitive and behavioural responses, fatigue severity, physical functioning and work/social adjustment. Hypothesis 2 was assessed using a repeated-measures analysis of covariance (RM-ANCOVA) to test for an interaction effect of time $\times$ group for fatigue severity, physical functioning and work/social adjustment, and a follow-up RM-ANCOVA, split by group and controlling for gender, to test for a significant treatment effect for the White British and BME groups. Partial eta-squared $\left(\eta_{P}^{2}\right)$ was the measure of effect size for the RM-ANCOVAs, and was categorised as small $(\leqslant 0.01)$, medium $(0.06)$ or large $(\geqslant 0.14)$. Group differences in change scores before and after treatment were tested using independent samples $t$-tests. RM-ANCOVAs, controlling for gender, were used in post-hoc analysis to test for an interaction effect of time ethnic subgroup for fatigue severity, physical functioning and work/social adjustment.

\section{Results}

\section{Participants}

The study sample included two groups, BME participants (19.0\%, $n=67)$ and White British participants $(81.0 \%, n=285)$. BME participants had multiple ethnicities: Black or Black British $(23.9 \%, n=16)$, Asian or Asian British $(16.4 \%, n=11)$, Caribbean $(7.5 \%, n=5)$, White European $(4.5 \%, n=3)$, East African Asian $(3.0 \%, n=2)$, Filipino $(3.0 \%, n=2)$, White and Asian $(3.0 \%$, $n=2)$, White and Black African $(3.0 \%, n=2)$, Arab $(1.5 \%$, $n=1)$, British Egyptian/mixed $(1.5 \%, n=1)$, Black Caribbean/ mixed $(1.5 \%, n=1)$, Black Caribbean and White $(1.5 \%, n=1)$,
Chinese $(1.5 \%, n=1)$, Ghanaian $(1.5 \%, n=1)$, Israeli/Jewish $(1.5 \%, n=1)$, Portuguese $(1.5 \%, n=1)$, Malaysian and Irish $(1.5 \%, n=1)$, mixed White Caribbean $(1.5 \%, n=1)$, Nigerian $(1.5 \%, n=1)$, Serbian $(1.5 \%, n=1)$, Southern European $(1.5 \%$, $n=1)$, Turkish Cypriot $(1.5 \%, n=1)$, White and Malaysian $(1.5 \%, n=1)$, White Irish $(1.5 \%, n=1)$, White South African $(1.5 \%, n=1)$, Black other $(1.5 \%, n=1)$, White other $(4.5 \%$, $n=3)$, mixed White other $(1.5 \%, n=1)$, mixed $(1.5 \%, n=1)$ and other ethnic group $(1.5 \%, n=1)$.

Table 1 shows the baseline demographic characteristics of both groups. There was a significantly higher proportion of females among the BME participants $(88.1 \%, n=59)$ than the White British participants $(71.2 \%, n=203), \chi^{2}(1,352)=8.08, P=0.005$. There were no significant group differences in duration of CFS, age, marital status, education, hours worked per week or work status.

\section{Attrition rate}

The total attrition rate in this study was $35.8 \%(n=126)$. Of these, $17.1 \%(n=60)$ dropped out during treatment $(81.7 \%$ White British, $n=49 ; 18.3 \%$ BME, $n=11$ ). A further $18.8 \%$ $(n=66)$ completed treatment but did not complete discharge questionnaires (72.7\% White British, $n=48 ; 27.3 \%$ BME, $n=18$ ).

Chi-square analysis and $t$-tests were conducted to test whether responders and non-responders differed in their baseline data. Chi-square analysis revealed a significant trend for females and those who were not working, to be less likely to respond/complete compared with males and those who were working, respectively $\left(\chi^{2}(1, n=352)=4.38, P=0.041, \chi^{2}(1, n=283)=8.63, P=0.004\right.$, respectively). There were no significant differences between responders and non-responders in other baseline measures, including ethnic group, fatigue severity, physical functioning and work/social adjustment; therefore, the risk of attrition bias was low.

\section{Between-group baseline comparisons}

As Table 2 shows, BME participants reported significantly higher baseline damage beliefs $(P=0.009)$, catastrophising $(P=0.024)$, all-or-nothing behaviour $(P=0.036)$ and avoidance/resting behaviour $(P=0.001)$. There were no differences between groups

\begin{tabular}{|c|c|c|c|}
\hline Characteristic & BME $(n=67)$ & White British $(n=285)$ & $P$ \\
\hline Age in years, mean (s.d.) & $40.79(10.50)$ & $38.18(11.65)$ & 0.094 \\
\hline \multicolumn{4}{|l|}{ Gender, \% } \\
\hline Male & 11.9 & 28.8 & 0.005 \\
\hline Female & 88.1 & 71.2 & \\
\hline \multicolumn{4}{|l|}{ Marital status, \% } \\
\hline Single & 47.8 & 42.7 & 0.060 \\
\hline Married & 35.8 & 49.8 & \\
\hline Divorced & 13.4 & 6.4 & \\
\hline Widowed & 3.0 & 1.1 & \\
\hline \multicolumn{4}{|l|}{ Educational qualification, \% } \\
\hline None & 0.0 & 4.7 & 0.250 \\
\hline Senior school & 24.6 & 26.4 & \\
\hline Polytechnic & 18.5 & 20.9 & \\
\hline University & 56.9 & 48.0 & \\
\hline \multicolumn{4}{|l|}{ Work status } \\
\hline Not working, \% & 64.2 & 62.7 & 0.873 \\
\hline Working, \% & 35.8 & 37.3 & \\
\hline Hours worked per week, mean (s.d.) & $18.43(16.69)$ & $17.49(17.40)$ & 0.725 \\
\hline
\end{tabular}




\begin{tabular}{|lcccc|}
\hline Table 2 Baseline clinical characteristics & & & & \\
Clinical data & Possible range & $\begin{array}{c}\text { BME, mean (s.d.) } \\
(n=67)\end{array}$ & $\begin{array}{c}\text { White British, mean (s.d.) } \\
(n=285)\end{array}$ & $P$ \\
\hline CFS duration (months) & - & $89.31(79.30)$ & $73.34(76.40)$ & 0.167 \\
\hline Fatigue severity & $0-33$ & $24.82(6.79)$ & $24.97(6.16)$ & 0.866 \\
\hline Physical functioning & $0-100$ & $44.39(25.99)$ & $49.19(25.33)$ & 0.168 \\
\hline Work/social adjustment & $0-40$ & $25.32(10.12)$ & $23.52(9.07)$ & 0.159 \\
\hline Damage beliefs & $0-20$ & $11.86(4.20)$ & $10.45(3.78)$ & 0.009 \\
\hline Fear avoidance & $0-24$ & $14.25(4.47)$ & $13.75(4.23)$ & 0.391 \\
\hline Catastrophising & $0-16$ & $8.94(3.70)$ & $7.84(3.56)$ & 0.024 \\
\hline Symptom focusing & $0-24$ & $14.45(5.22)$ & $13.43(5.04)$ & 0.148 \\
\hline Embarrassment avoidance & $0-24$ & $13.50(5.18)$ & $12.08(5.62)$ & 0.062 \\
\hline All-or-nothing behaviour & $0-20$ & $10.83(5.03)$ & $9.46(4.76)$ & 0.036 \\
\hline Avoidance/resting behaviour & $0-32$ & $15.67(6.38)$ & $12.97(5.59)$ & 0.001 \\
\hline $\begin{array}{l}\text { BME, Black and minority ethnic; CFS, chronic fatigue syndrome. } \\
\text { a. Higher score indicates better physical functioning. }\end{array}$ & & & \\
\hline
\end{tabular}

in fatigue severity, physical functioning, work/social adjustment, fear avoidance, symptom focusing or embarrassment avoidance.

\section{Between-group outcome comparisons}

Fatigue severity outcomes

The RM-ANCOVA, controlling for gender, revealed a main effect of time on fatigue severity $\left(F(1,214)=15.59, P<0.001, \eta_{P}^{2}=0.07\right)$, but no interaction effect of time $\times$ group $(F(1,214)=0.51$, $P=0.475, \eta_{P}^{2}=0.00$ ). To follow up the main effect of time, pairwise comparisons revealed a significant treatment effect for both groups $(P<0.001)$. Table 3 shows that fatigue severity scores decreased by 8.34 points (95\% CI $5.10-11.57$ ) in BME participants, and by 7.37 points (95\% CI 6.17-8.57) in White British participants. There were no significant differences in change scores between BME and White British participants $(t(198)=-0.28, P=0.777)$.

\section{Physical functioning outcomes}

The RM-ANCOVA, controlling for gender, revealed a main effect of time on physical functioning $(F(1,209)=4.38, P=0.038$, $\left.\eta_{P}^{2}=0.02\right)$, but no interaction effect of time $\times$ group $\left(F(1,209)=0.32, P=0.574, \eta_{P}^{2}=0.00\right)$. To follow up the main effect of time, pairwise comparisons revealed a significant treatment effect for both groups $(P<0.001)$. Table 3 shows that physical functioning scores increased by 11.39 points (95\% CI 5.31-17.46) in BME participants and by 9.46 points (95\% CI 6.68-12.24) in White British participants. There were no significant differences in change scores between BME and White British participants $(t(190)=0.23, P=0.821)$.

\section{Work/social adjustment outcomes}

The RM-ANCOVA, controlling for gender, revealed a main effect of time on work/social adjustment $(F(1,215)=11.21, P=0.001$, $\left.\eta_{P}^{2}=0.05\right)$, but no interaction effect of time $\times$ group
$\left(F(1,215)=0.07, P=0.790, \eta_{P}^{2}=0.00\right)$. To follow up the main effect of time, pairwise comparisons revealed a significant treatment effect for both groups $(P<0.001)$. Table 3 shows that Work and Social Adjustment Scale scores decreased by 6.01 points $(95 \%$ CI 3.87-8.16) in BME participants and 5.75 points (95\% CI 4.64-6.87) in White British participants. There were no significant differences in change scores between BME and White British participants $(t(189)=-0.96, P=0.339)$.

\section{Post-hoc analysis: BME subgroups}

The BME group was divided into subgroups: Black (43.3\%, $n=29)$, Asian (29.9\%, $n=20)$ and other ethnic minority (26.9\%, $n=18)$. An RM-ANCOVA showed no significant interaction effect of time $\times$ ethnic subgroup for fatigue severity $\left(F(1,212)=1.24, \quad P=0.296, \quad \eta_{P}^{2}=0.02\right), \quad$ physical functioning $\left(F(1,207)=0.20, P=0.896, \eta_{P}^{2}=0.00\right)$ or work/social adjustment $\left(F(1,213)=1.58, P=0.194, \eta_{P}^{2}=0.02\right)$. See online Fig. DS1 for mean pre- and post-outcome scores for White British and BME subgroup participants.

\section{Discussion}

\section{Main findings}

Contrary to expectations, and to a popular view that CBT is culturally biased and suitable only for people of European or North American cultural origins, ${ }^{12}$ the present study shows that CBT is effective for both BME and White British patients. This study is, therefore, the first to indicate that CBT is an effective treatment for CFS in a diverse group of BME patients presenting to CFS services, and consequently challenges the notion that CBT requires cultural adaptation for use in English-speaking BME groups.

Table 3 Changes in scores of fatigue severity, physical functioning and work/social adjustment before and after CBT

\begin{tabular}{|c|c|c|c|c|c|c|}
\hline \multirow[b]{2}{*}{ Variable } & \multicolumn{3}{|c|}{ BME } & \multicolumn{3}{|c|}{ White British } \\
\hline & $\begin{array}{c}\text { Pre-CBT } \\
\text { mean (s.d.) }\end{array}$ & $\begin{array}{l}\text { Post-CBT } \\
\text { mean (s.d.) }\end{array}$ & $\begin{array}{c}\text { Change } \\
\text { mean }\left(\eta_{P}^{2}\right)\end{array}$ & $\begin{array}{c}\text { Pre-CBT } \\
\text { mean (s.d.) }\end{array}$ & $\begin{array}{l}\text { Post-CBT } \\
\text { mean (s.d.) }\end{array}$ & $\begin{array}{l}\text { Change } \\
\text { mean }\left(\eta_{P}^{2}\right)\end{array}$ \\
\hline Fatigue severity & $25.57(6.40)$ & $17.23(9.74)$ & $8.34 *(0.44)$ & 24.96 (5.94) & $17.59(8.49)$ & $7.37 *(0.45)$ \\
\hline Physical functioninga & $44.40(25.04)$ & $55.78(28.16)$ & $11.39 *(0.31)$ & $51.20(25.16)$ & $60.66(28.68)$ & $9.46 *(0.20)$ \\
\hline Work/social adjustment & $26.90(9.36)$ & 20.89 (12.18) & $6.01 *(0.47)$ & $23.40(8.50)$ & $17.65(10.67)$ & $5.75 *(0.37)$ \\
\hline
\end{tabular}




\section{Baseline comparisons}

It was found that BME participants improved despite exhibiting significantly higher baseline scores relating to catastrophising, damage beliefs, all-or-nothing behaviour and avoidance/resting behaviour compared with White British participants, suggesting that these should not act as a barrier to good post-treatment outcomes. Higher levels of catastrophising exhibited by BME participants may be related to lower levels of optimism within this group $^{5}$ and exacerbated by difficulties that BME patients face in diagnosis and treatment. For example, language may be a barrier to the discussion of fatigue in some BME groups, with some patients less able to adequately describe their symptoms or understand their general practitioner. ${ }^{10}$ Avoidance/resting behaviour, which is associated with poorer general health, may be related to social strain, poverty or racial discrimination and may be more commonly experienced by BME groups. ${ }^{31}$ For example, some BME groups psychologically disengage to maintain self-esteem when faced with negative stereotyping and prejudice. ${ }^{32}$

Higher levels of all-or-nothing behaviour might be related to lower treatment expectations in BME groups, ${ }^{33}$ resulting in over-extension when feeling well and resultant periods of resting. Finally, the higher levels of damage beliefs exhibited by BME groups might result from differences in illness attributions made by the two study groups. For example, Bayliss and colleagues ${ }^{10}$ found that the perception among BME community leaders was that CFS patients within this group were more likely to hold a biomedical model of their illness. It could be argued that emphasising the physical aspects of illness, rather than non-specific symptoms such as fatigue, loss of concentration or problems with sleep, may amplify the belief that these symptoms indicate structural damage or injury. Nonetheless, the significant post-treatment improvement in outcomes suggest that these unhelpful cognitive and behavioural responses to symptoms at baseline were successfully targeted and modified during treatment, providing further support for the effectiveness of CBT in both BME and White British groups.

\section{Previous literature}

Contrary to what is seen in previous literature, ${ }^{13-16}$ we found no ethnic group differences in treatment outcome. However, in contrast to past work, ${ }^{15,16}$ treatment in our study was restricted to $\mathrm{CBT}$ and was delivered by specialist CBT practitioners. Moreover, our findings were not restricted by a small sample size, or by a lack of a priori hypotheses, as has been the case in previous CBT studies. ${ }^{13,14}$ Furthermore, although ethnic differences in CFS severity have been found, ${ }^{4-8}$ this may have been due to the variety of CFS-related conditions previously investigated (such as 'CFS-like' illnesses, ${ }^{7,8}$ and 'chronic fatigue ${ }^{4,6}$ ). The heterogeneity of the CFS criteria used in different studies ${ }^{5}$ can also affect both treatment and the conclusions drawn from research. ${ }^{34}$ To our knowledge, this study is the first to use UK criteria ${ }^{1}$ to explore ethnic group differences in CFS severity and CBT treatment outcomes.

\section{Strengths and limitations}

As there are limitations to this study, these results should be interpreted with caution. First, the Chronic Fatigue Research and Treatment Unit is situated in the London borough of Southwark, where $46 \%$ of the population is from a BME background. ${ }^{35}$ This is higher than the proportion of referrals seen in the service and in the present study (19\% from a BME background) and may be indicative of referral or selection bias. That is, those attending are more likely to be similar to White British patients, have a positive attitude to psychological therapies and feel less CFS-related stigma. Therefore, culturally adapted interventions may be required for those not referred to this setting or only treated in primary care.

Second, the BME group consisted of several subgroups, with individuals from a wide variety of cultural and ethnic backgrounds. Because of the low numbers within each subgroup, meaningful pre- and post-test assessments of the subgroups could not be undertaken and the assumptions of statistical tests would not hold. However, post-hoc tests of variations between three subgroups - Black, Asian, and other ethnic minority groups revealed no significant differences in outcomes.

Third, multiple testing increased the risk of type 1 statistical error. Multiple test adjustments such as Bonferroni corrections were not used, as these can be overly conservative and can be detrimental to statistical inference. ${ }^{36}$ Finally, this study lacked a control group, therefore it is not possible to eliminate alternative explanations for experimental results.

A strength of this study is that CFS was diagnosed using well-defined, operationalised criteria ${ }^{1}$ after a thorough clinically driven biopsychosocial assessment. Furthermore, all participants completed identical questionnaires at baseline and discharge, which were reliable and had been validated in CFS populations, minimising information bias. There were no differences between responders and non-responders in baseline fatigue severity, physical functioning, work/social adjustment or ethnic-group status, indicating that the sample was minimally affected by attrition bias. The treatment dropout rate was also in line with a systematic review of $\mathrm{CBT}$ for $\mathrm{CFS},{ }^{37}$ suggesting that treatment was well tolerated.

In conclusion, this study is the first to indicate that CBT is effective in a group of patients from diverse BME backgrounds presenting to a CFS service. These findings are in contrast to the theory that CBT is culturally biased and suggest that culturally adapted CBT might not be necessary for some English-speaking BME patients. Results also indicate that any unhelpful baseline cognitions and behaviours exhibited by BME patients should not hinder good post-CBT outcomes. A larger, randomised controlled trial is required to confirm the effectiveness of CBT for BME patients, as this is considered the most rigorous method for evaluating treatments. Even in trials, however, there are difficulties recruiting sufficient participants from BME groups, so the recommendation is often to ensure representation of BME patients in the trial arms match their representation in the population, rather than recruit by specific BME subgroup. Future research could investigate whether CBT treatment for CFS is sensitive to cultural needs by using a qualitative analysis to explore the experiences of community-based individuals who do not seek help.

\footnotetext{
Tom Ingman, MSC, King's College London, Institute of Psychiatry, Psychology and Neuroscience, London, UK' Sheila Ali, MSC, Chronic Fatigue Research and Treatment Unit, South London and Maudsley NHS Foundation Trust, London, UK; Kamaldeep Bhui, MD, FRCPsych, Wolfson Institute of Preventive Medicine, Barts and The London School of Medicine and Dentistry, Queen Mary University of London, London, UK; Trudie Chalder, PhD, King's College London, Institute of Psychiatry, Psychology and Neuroscience, London, UK.

Correspondence: Professor Trudie Chalder, King's College London, Weston Education Centre, Cutcombe Road, London SE5 9RJ, UK. Email: trudie.chalder@ kcl.ac.uk

First received 5 May 2015, accepted 12 Jun 2015
}

\section{Funding}

TC. receives salary support from the National Institute for Health Research (NIHR), Mental Health Biomedical Research Centre at South London and Maudsley National Health Service Health Biomedical Research Centre at South London and Maudsley National Health Service
Foundation Trust and King's college London. The views expressed are those of the authors and not necessarily those of the NHS, or the NIHR. 


\section{Acknowledgements}

We thank the patients who took part in this study. We also thank Ms Suzanne Roche Ms Barbara Bowman, Dr Mary Burgess, Dr Antonia Dittner, Dr Caroline Stokes, Dr Anna Hutchinson and Ms Sue Wilkins for delivering CBT treatment and for collecting data.

\section{References}

1 Sharpe MC, Archard LC, Banatvala JE, Borysiewicz LK, Clare AW, David A, et al. A report - chronic fatigue syndrome: guidelines for research. $J R$ SOC Med 1991; 84: 118-21.

2 Reyes M, Gary Jr H, Dobbins J, Randall B, Steele L, Fukuda K, et al. Surveillance for chronic fatigue syndrome - four US cities, September 1989 through August 1993. MMWR CDC Surveill Summ 1997; 46: 1-13.

3 Dinos S, Khoshaba B, Ashby D, White PD, Nazroo J, Wessely S, et al. A systematic review of chronic fatigue, its syndromes and ethnicity: prevalence, severity, co-morbidity and coping. Int J Epidemiol 2009; 38 1554-70.

4 Jason LA, Jordan KM, Richman JA, Rademaker AW, Huang CF, Mccready W, et al. A community-based study of prolonged fatigue and chronic fatigue. J Health Psychol 1999; 4: 9-26.

5 Jason L, Taylor R, Kennedy C. Chronic fatigue syndrome. Eval Health Prof 2000; 23: 243-63.

6 Jason LA, Taylor RR, Kennedy CL, Jordan K, Huang C, Torres-Harding SR, et al. A factor analysis of chronic fatigue symptoms in a community-based sample. Soc Psychiatry Psychiatr Epidemiol 2002; 37: 183-9.

7 Song S, Jason LA, Taylor RR. The relationship between ethnicity and fatigue in a community-based sample. J Gend Cult Health 1999; 4: 255-68.

8 Song S, Jason LA, Taylor R, Torres-Harding SR, Helgerson J, Witter E. Fatigue severity among African Americans: gender and age interactions. J Black Psychol 2002; 28: 53-65.

9 Njoku M, Jason L, Torres-Harding S. The relationships among coping styles and fatigue in an ethnically diverse sample. Ethn Health 2005; 10: 263-78.

10 Bayliss K, Riste L, Fisher L, Wearden A, Peters S, Lovell K, et al. Diagnosis and management of chronic fatigue syndrome/myalgic encephalitis in black and minority ethnic people: a qualitative study. Prim Health Care Res Dev 2014; 15: $143-55$

11 White PD, Goldsmith KA, Johnson AL, Potts L, Walwyn R, DeCesare JC, et al. Comparison of adaptive pacing therapy, cognitive behaviour therapy, graded exercise therapy, and specialist medical care for chronic fatigue syndrome (PACE): a randomised trial. Lancet 2011; 377: 823-36.

12 Kantrowitz RE, Ballou MA. A feminist critique of cognitive-behavioral therapy. In Personality and Psychopathology: Feminist Reappraisals (eds LS Brown and M Ballou): 70-87. Guildord, 1992.

13 Markowitz JC, Spielman LA, Sullivan M, Fishman B. An exploratory study of ethnicity and psychotherapy outcome among HIV-positive patients with depressive symptoms. J Psychother Pract Res 2000; 9: 226-31.

14 Schneiderman N, Saab PG, Catellier DJ, Powell LH, DeBusk RF, Williams RB et al. Psychosocial treatment within sex by ethnicity subgroups in the Enhancing Recovery in Coronary Heart Disease clinical trial. Psychosom Med 2004; 66: 475-83.

15 Rathod S, Kingdon D, Smith P, Turkington D. Insight into schizophrenia: the effects of cognitive behavioural therapy on the components of insight and association with sociodemographics - data on a previously published randomised controlled trial. Schizophr Res 2005; 74: 211-9.

16 Boydell J, Morgan C, Dutta R, Jones B, Alemseged F, Dazzan P, et al. Satisfaction with inpatient treatment for first-episode psychosis among different ethnic groups: A report from the UK AESOP study. Int I SOC Psychiatry 2012; 58: 98-105.

17 Rathod S, Phiri P, Harris S, Underwood C, Thagadur M, Padmanabi U, et al Cognitive behaviour therapy for psychosis can be adapted for minority ethnic groups: a randomised controlled trial. Schizophr Res 2013; 143: 319-26.

18 Chowdhary N, Jotheeswaran AT, Nadkarni A, Hollon SD, King M, Jordans MJ, et al. The methods and outcomes of cultural adaptations of psychological treatments for depressive disorders: a systematic review. Psychol Med 2013; 44: 1131-46.

19 National Institute for Health and Clinical Excellence. Chronic Fatigue Syndrome/Myalgic Encephalomyelitis (or Encephalopathy): Diagnosis and Management of CFS/ME in Adults and Children (CG53). NICE, 2007.

20 Brooks SK, Rimes KA, Chalder T. The role of acceptance in chronic fatigue syndrome. J Psychosom Res 2011; 71; 411-5.

21 Chalder T, Berelowitz G, Pawlikowska T, Watts L, Wessely S, Wright D, et al. Development of a fatigue scale. J Psychosom Res 1993; 37; 147-53.

22 Cella $M$, Chalder T. Measuring fatigue in clinical and community settings. J Psychosom Res 2010; 69: 17-22.

23 Ware Jr JE, Sherbourne CD. The MOS 36-item short-form health survey (SF36): I. Conceptual framework and item selection. Med Care 1992; 30: 473-83.

24 Buchwald D, Manson SM, Pearlman T, Umali J, Kith P. Race and ethnicity in patients with chronic fatigue. J Chronic Fatigue Syndr 1996; 2: 53-66.

25 Mundt JC, Marks IM, Shear MK, Greist JM. The Work and Social Adjustment Scale: a simple measure of impairment in functioning. Br J Psychiatry 2002; 180; $461-4$.

26 Cella M, Sharpe M, Chalder T. Measuring disability in patients with chronic fatigue syndrome: reliability and validity of the Work and Social Adjustment Scale. J Psychosom Res 2011; 71: 124-8.

27 Cella M, White PD, Sharpe M, Chalder T. Cognitions, behaviours and co-morbid psychiatric diagnoses in patients with chronic fatigue syndrome. Psychol Med 2012; 43: 375-80.

28 Skerrett TN, Moss-Morris RE. Fatigue and social impairment in multiple sclerosis: the role of patients' cognitive and behavioral responses to their symptoms. J Psychosom Res 2006; 61: 587-93.

29 Knudsen AK, Henderson M, Harvey SB, Chalder T. Long-term sickness absence among patients with chronic fatigue syndrome. Br J Psychiatry 2011; 199: 430-1.

30 Stahl D, Rimes K, Chalder T. Mechanisms of change underlying the efficacy of cognitive behaviour therapy for chronic fatigue syndrome in a specialist clinic: a mediation analysis. Psychol Med 2014; 44: 1331-44.

31 Erens B, Primatesta P, Prior G. Health Survey for England: the Health of Minority EthnicGroups. TSO (The Stationery Office), 2001.

32 Major B, Schmader T. Coping with stigma through psychological disengagement. In Prejudice: The Target's Perspective (eds JK Swim, C Stangor): 219-41. Academic Press, 1998.

33 Collins J, Hyer L. Treatment expectancy among psychiatric inpatients. J Clin Psychol 1986; 42: 562-9.

34 Ax S, Gregg V, Jones D. Coping and illness cognitions: chronic fatigue syndrome. Clin Psychol Rev 2001; 21: 161-82.

35 Office for National Statistics. 2011 Census: Aggregate Data (England and Wales). UK Data Service Census Support, 2011.

36 Perneger TV. What's wrong with Bonferroni adjustments. BMJ 1998; 316 $1236-8$

37 Malouff JM, Thorsteinsson EB, Rooke SE, Bhullar N, Schutte NS. Efficacy of cognitive behavioral therapy for chronic fatigue syndrome: a meta-analysis. Clin Psychol Rev 2008; 28: 736-45. 